Document downloaded from:

http://hdl.handle.net/10251/47801

This paper must be cited as:

Gomis, O.; Sans, J. A.; Lacomba-Perales, R.; et ál..(2012). Complex high-pressure polymorphism of barium tungstate. Physical Review B. 86:54121-1-54121-10. doi:10.1103/PhysRevB.86.054121.

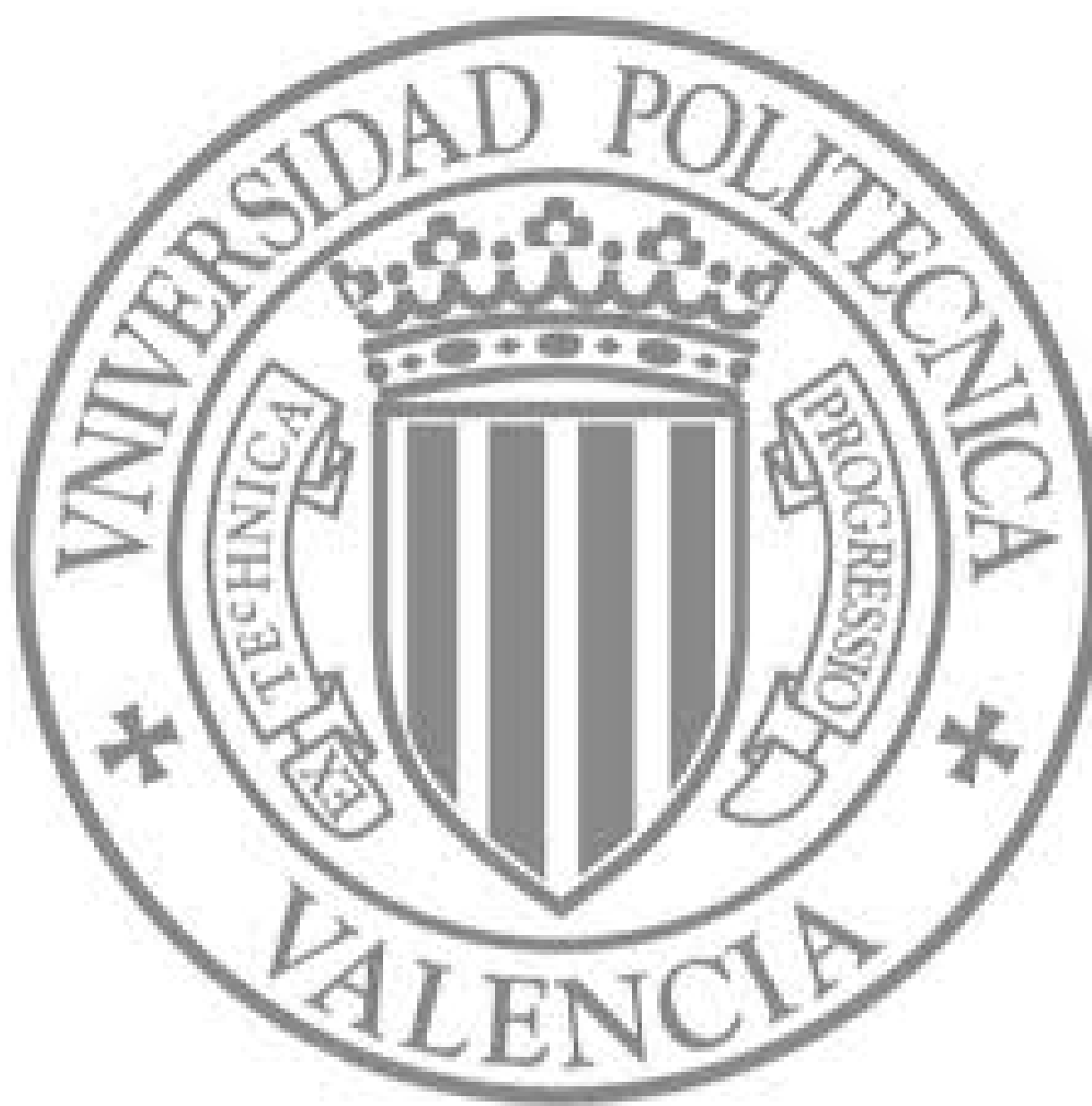

The final publication is available at

http://journals.aps.org/prb/pdf/10.1103/PhysRevB.86.054121

Copyright American Physical Society 


\title{
Complex high-pressure polymorphism of barium tungstate
}

\author{
O. Gomis ${ }^{1, *}$, J.A. Sans ${ }^{2,3}$, R. Lacomba-Perales ${ }^{2}$, D. Errandonea ${ }^{2}$, Y. Meng ${ }^{4}$, J. C. \\ Chervin $^{5}$, and A. Polian ${ }^{5}$
}

\begin{abstract}
${ }^{1}$ Centro de Tecnologías Físicas, MALTA Consolider Team, Universitat Politècnica de València, 46022 València, Spain

${ }^{2}$ Departamento de Física Aplicada-ICMUV, Universidad de Valencia, MALTA Consolider Team, Edificio de Investigación, C/Dr. Moliner 50, 46100 Burjassot, Spain

${ }^{3}$ Instituto de Diseño para la Fabricación y Producción Automatizada, MALTA Consolider Team, Universitat Politècnica de València, 46022 València, Spain

${ }^{4}$ HPCAT, Carnegie Institution of Washington, Building 434E, 9700 South Cass Avenue, Argonne, Illinois 60439, USA

${ }^{5}$ Institut de Minéralogie et de Physique des Milieux Condensés, Université Pierre et Marie Curie-Sorbonne Universités, CNRS UMR 7590, 140 rue de Lourmel, F-75015 Paris, France
\end{abstract}

\begin{abstract}
We have studied $\mathrm{BaWO}_{4}$ under compression at room temperature by means of x-ray diffraction and Raman spectroscopy. When compressed with neon as pressuretransmitting medium (quasi-hydrostatic conditions), we found that $\mathrm{BaWO}_{4}$ transforms from its low-pressure tetragonal structure into a much denser monoclinic structure. This result confirms our previous theoretical prediction based on ab initio calculations that the scheelite to $\mathrm{BaWO}_{4}$-II transition occurs at room temperature if kinetic barriers are suppressed by pressure. However, our experiment without any pressure-transmitting medium has resulted in a phase transition to a completely different structure, suggesting non-hydrostaticity may be responsible for previously reported rich polymorphism in $\mathrm{BaWO}_{4}$. The crystal structure of the low- and high-pressure phases from the quasihydrostatic experiments has been Rietveld refined. Additionally, for the tetragonal phase the effects of pressure on the unit-cell volume and lattice parameters is discussed.
\end{abstract}


Finally, the pressure evolution of the Raman modes of different phases is reported and compared with previous studies.

Keywords: high pressure, barium tungstate, polymorphism, x-ray diffraction, Raman scattering.

PACS number(s): 62.50.-p, 61.50.Ks, 61.05.cp, 63.20.dd

\section{Introduction}

Barium tungstate $\left(\mathrm{BaWO}_{4}\right)$ is a material that crystallize in the tetragonal

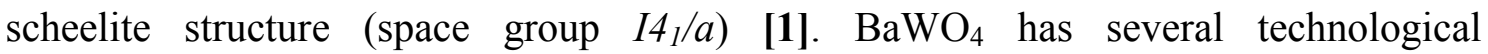
applications and has been extensively studied under compression [2]. However, its complex polymorphism remains the subject of continuous study [3-11]. At ambient to moderate pressures and room temperature (RT), there are two different forms of $\mathrm{BaWO}_{4}$ which are built up of $\mathrm{WO}_{4}$ tetrahedra and $\mathrm{BaO}_{8}$ dodecahedra. The first is the scheelitetype and the second is a monoclinic fergusonite-type (space group $I 2 / a$ ) which is a highpressure phase. At higher pressures and high temperatures, a denser form containing $\mathrm{WO}_{6}$ octahedra occurs $[\mathbf{9}, \mathbf{1 2}, \mathbf{1 3}]$. This polymorph is monoclinic and is named $\mathrm{BaWO}_{4}-$ II (space group $P 2_{1} / n$ ). It has been discussed that there are large kinetic barriers associated with the transformation from the scheelite-type to the $\mathrm{BaWO}_{4}$-II structure [9] and thus the application of high temperature (HT) is needed to overcome the kinetics barriers. As a consequence, the fergusonite-type phase can be observed in between the other two phases at RT. In addition, amorphization has been reported after the $\mathrm{BaWO}_{4^{-}}$ II phase beyond $47 \mathrm{GPa}$ [9]. Recently, it has been shown that non hydrostatic stresses could strongly influence the high-pressure (HP) structural sequence of $\mathrm{BaWO}_{4}$. It has been also debated, whether the RT post-fergusonite monoclinic phase has the same 
crystal structure as that of the HP-HT BaWO 4 -II phase [3-6]. Therefore, it is clear that further efforts are needed to elucidate the complex HP behavior of $\mathrm{BaWO}_{4}$. Here, we report HP x-ray diffraction (XRD) and Raman studies of $\mathrm{BaWO}_{4}$. Raman studies were performed under quasi-hydrostatic conditions using neon $(\mathrm{Ne})$ as pressure-transmitting medium up to $45 \mathrm{GPa}$. XRD studies were performed up to $17.3 \mathrm{GPa}$ under similar conditions and also under highly non hydrostatic conditions to check the influence of non hydrostaticity. The present results are compared with previous results obtained under a large variety of experimental conditions, showing that at least four different phases of $\mathrm{BaWO}_{4}$ can be obtained at the same pressure. Present experiments also prove that under quasi-hydrostatic conditions $\mathrm{BaWO}_{4}$ transform at $\mathrm{RT}$ directly from scheelite to $\mathrm{BaWO}_{4}$-II. The results here reported are relevant for the understanding of the highpressure behavior of other tungstates with technological applications like $\mathrm{PbWO}_{4}[\mathbf{9}$, 14,15]. They are also calling for the reexamination of high-pressure studies on different binary and ternary oxides since apparently non-hydrostaticity could strongly influence the series of crystalline structures appearing under compression.

\section{Experimental Details}

Samples used in the present experiments were obtained from a high-purity $\mathrm{BaWO}_{4}$ single crystal, which was grown with the Czochralski method starting from raw powders having $5 N$ purity [9]. It was confirmed by XRD and Raman spectroscopy that the crystal has only one phase with the scheelite structure. For the HP Raman experiment, a $10-\mu \mathrm{m}$-thick plate was cleaved from a crystal and loaded in a $300-\mu \mathrm{m}$ culet diamond-anvil cell (DAC) together with a few ruby spheres of about $2 \mu \mathrm{m}$ in diameter. Ne was used as pressure-transmitting medium (PTM) and pressure was determined by the ruby fluorescence technique [16]. According to Klotz et al. [17] Ne provides better hydrostic conditions than any of the pressure media used before to study 
$\mathrm{BaWO}_{4}$ showing the first signs of non-hydrostaticity only beyond $15 \mathrm{GPa}$. Since the phase transition of main interest for this work occurs below $10 \mathrm{GPa}$, the use of $\mathrm{Ne}$ is a good choice to obtain quasi-hydrostatic conditions.

Room-temperature Raman experiments were performed in the backscattering geometry using the $514.5 \mathrm{~nm}$ line of an $\mathrm{Ar}^{+}$-ion laser with a power of less than $100 \mathrm{~mW}$ before the DAC to avoid sample heating. Laser heating of the sample by the $514.5 \mathrm{~nm}$ laser is negligible in the studied pressure range because the laser energy is always below the band gap of $\mathrm{BaWO}_{4}$ in the pressure range covered by experiments [4]. A Mitutoyo 20X long working distance objective was employed for focusing the laser on the sample and for collecting the Raman spectra. The scattered light was analyzed with a JobinYvon T64000 triple spectrometer equipped with a confocal microscope in combination with a liquid-nitrogen cooled multichannel charge-coupled device (CCD) detector. The spectral resolution was better than $1 \mathrm{~cm}^{-1}$. For $\mathrm{HP}$ x-ray diffraction experiments, samples were prepared as fine ground powders from the single crystal.

High-pressure angle-dispersive XRD measurements were carried out in a 400- $\mu \mathrm{m}$ culet DAC. Powder samples were loaded together with a ruby chip used for pressure determination. Two independent experiments were carried out, one using $\mathrm{Ne}$ as pressure-transmitting medium and the other without PTM. XRD experiments were performed at the 16-IDB beamline of the HPCAT facility at the Advanced Photon Source (APS). Monochromatic synchrotron radiation at $\lambda=0.368018 \AA$ was used for data collection on a Mar345 image-plate detector. The x-ray beam was focused down to 5 x $5 \mu \mathrm{m}^{2}$ using Kickpatrick-Baez mirrors. The diffraction images were integrated and corrected for distortions using FIT2D [18] to yield intensity versus $2 \theta$ diagrams. Indexing, structure solution, and refinements were performed using the UNITCELL [19], GSAS [20,21], and POWDERCELL [22] program packages. 


\section{Results and Discussion}

\section{A. XRD experiments}

Figure 1 shows a selection of diffraction patterns collected in the two XRD experiments. In Fig. 1(a), it can be seen that XRD data collected under $\mathrm{Ne}$ can be assigned to the scheelite structure up to $7.6 \mathrm{GPa}$. The fact that Bragg peaks do not broaden upon compression in this experiment supports the assumption that it was performed under quasi-hydrostatic conditions. The results of the Rietveld refinement obtained for this structure at 1.0 and 7.6 GPa are shown in the figure together with the experimental data. In addition to the unit-cell parameters, we also refined the oxygen positions, which can be obtained because the positions of $\mathrm{W}$ and $\mathrm{Ba}$ (which dominates the x-ray scattering) are fixed by the structural symmetry of scheelite. Since the occupancy and the atomic displacement factors are correlated and more sensitive to background subtraction than positional parameters, they were constrained to 1 and $B=$ $0.5 \AA^{2}$, where $B$ is the overall displacement factor, to reduce the number of free parameters used in the refinement. Table I provides the structural parameters obtained from the Rietveld refinements at 1.0 and $7.6 \mathrm{GPa}$ using $\mathrm{Ne}$ as PTM and the ambient pressure structural parameters obtained from neutron-diffraction experiments [1]. The small residuals are consistent with the assignation of the scheelite structure to $\mathrm{BaWO}_{4}$ from 1 bar to $7.6 \mathrm{GPa}$. At this pressure, in addition to the Bragg reflections of the sample five peaks assigned to the solidification of $\mathrm{Ne}$ are detected. The atomic positions obtained from the refinement at $1.0 \mathrm{GPa}$ are similar to the ambient pressure parameters [1] and those previously reported at $1 \mathrm{GPa}$ [9]. At 7.6 GPa, we found a reduction of unit-cell parameters and small change of the oxygen positions. The observed changes for the oxygen positions are consistent with those obtained from neutron-diffraction 
experiments [8], which are more accurate to determine the positions of atoms with low atomic numbers like oxygen.

Upon further compression, at $9.4 \mathrm{GPa}$, the XRD pattern of $\mathrm{BaWO}_{4}$ completely changes indicating the occurrence of a structural phase transition. The determination of the crystal structure of the HP phase will be discussed after describing the second experiment and the effects of pressure on the scheelite structure within its range of stability. In Fig. 1(b), it can be seen that $\mathrm{BaWO}_{4}$ remains in the scheelite structure up to 7.2 $\mathrm{GPa}$ when no pressure-transmitting medium is used for the experiments. Upon further compression, changes associated to a phase transition take place at $8.6 \mathrm{GPa}$. The HP phase remains stable up to the maximum pressure reached in the experiment $(17.3$ GPa). The phase transition is reversible as can be seen in the XRD pattern collected after pressure was released to 1 bar, which can be unequivocally assigned to the scheelite phase. Another important fact that cannot be left without being commented is that XRD patterns of the HP phase are different in the two experiments, suggesting that different structures are stabilized at the same pressure as a consequence of nonhydrostaticity.

From the Rietveld refinements, we obtained (from both set of experiments) the pressure evolution of the lattice parameters and unit-cell volume of the low-pressure scheelite phase. Results are summarized in Fig. 2. From the pressure-volume curves, we obtained the equation of state (EOS) parameters using a third-order Birch-Murnaghan EOS [23]. In Table II, the bulk modulus $\left(\mathrm{B}_{0}\right)$, its pressure derivative $\left(\mathrm{B}_{0}{ }^{\prime}\right)$, and the atomic volume at zero pressure $\left(\mathrm{V}_{0}\right)$ are summarized and compared with those of previous studies. In the experiment performed with Ne as PTM the EOS fit was done by assuming $\mathrm{B}_{0}{ }^{\prime}=4$ due to the small dataset. However, in our XRD experiment without pressure-transmitting medium a value of $\mathrm{B}_{0}{ }^{\prime}=4(2)$ was obtained after the EOS fit. 
Therefore, in order to better compare with earlier experiments, previously reported data were fit again using $\mathrm{B}_{0}{ }^{\prime}=4$. In Table II, it can be seen that the bulk modulus decreases following the sequence No PTM $>$ silicone oil $\cong$ methanol-ethanol-water $>$ Ne. This indicates that non hydrostaticity causes a reduction of bulk compressibility as has been already found in other compounds [24 - 27]. The differences can be as large as $30 \%$ (see Table II). Differences are also detected for the axial compressibilities showing the silicone-oil and methanol-ethanol-water an intermediate behavior between the two limit cases here studied.

Fig. 2 and Table II also show that for the scheelite phase the compressibility along the $c$ axis is larger than that along the $a$ axis, which causes a reduction of the $c / a$ ratio under stress. This observation extends to other scheelites and can be related to the fact that when pressure is applied to the scheelite structure the $\mathrm{WO}_{4}$ tetrahedra remain essentially undistorted while the volume of the $\mathrm{BaO}_{8}$ bisdisphenoids is largely reduced [2]. Thus, the $a$ axis is expected to be less compressible than the $c$ axis because the $\mathrm{WO}_{4}$ units are directly aligned along the $a$ axis whereas along the $c$ axis there is a $\mathrm{Ba}$ cation between the $\mathrm{WO}_{4}$ tetrahedra [1]. It is interesting to note that the ratio of axial compressibilities is also affected by non hydrostaticity (see Table II). Consequently, non hydrostaticity not only reduces the bulk compressibility, but also affects the behavior of the axial ratio. Under quasi-hydrostatic conditions, c/a is reduced from 2.27 at $1.0 \mathrm{GPa}$ to 2.22 at $7.6 \mathrm{GPa}$, approaching the value corresponding to an ideal close packing.

From the present XRD results we further obtain information on bond compressibility (see Fig. 2d). For scheelite $\mathrm{BaWO}_{4}$, the first-neighbors $\mathrm{W}-\mathrm{O}$ distances are more rigid than the $\mathrm{Ba}-\mathrm{O}$ ones. In particular, under quasi-hydrostatic conditions, the short W-O distance decreases from $1.779 \AA$ at $1.0 \mathrm{GPa}$ to $1.719 \AA$ at $7.6 \mathrm{GPa}$ and the average $\mathrm{Ba}-\mathrm{O}$ distance decreases from $2.753 \AA$ at $1.0 \mathrm{GPa}$ to $2.654 \AA$ at $7.6 \mathrm{GPa}$. The 
bond compressibility for the short W-O distance is estimated as 5.0(3) $10^{-3} \mathrm{GPa}^{-1}$ and for the average $\mathrm{Ba}-\mathrm{O}$ distance 5.5(4) $10^{-3} \mathrm{GPa}^{-1}$. A similar qualitative behavior is found under different pressure media being always the W-O short distance at least $10 \%$ less compressible than the $\mathrm{Ba}-\mathrm{O}$ distance. On the other hand, we have found that under quasi-hydrostatic conditions the long W-O distance (second neighbor distance) is more compressible than the first neighbor distances with a cation-oxygen compressibility of 7.6(9) $10^{-3} \mathrm{GPa}^{-1}$. Ab initio calculations also report the long W-O distance as the most compressible one [9] as can be seen in Fig 2d.

As a consequence of the facts above described polyhedral compressibility of $\mathrm{BaO}_{8}$ dodecahedra and $\mathrm{WO}_{4}$ tetrahedra are different. This fact added to the larger volume of the dodecahedra makes the $\mathrm{BaO}_{8}$ units to account for most of the crystal compression as described above. To illustrate it, we have calculated the pressure evolution of both polyhedral units for the $\mathrm{Ne}$ experiment. Polyhedral volumes were obtained using DRAWxtl [28]. Results are summarized in Fig. 3 where it can be seen that the volume change of $\mathrm{BaO}_{8}$ units is larger than that of $\mathrm{WO}_{4}$ tetrahedra. It is interesting to note that temperature also basically affects only the volume of dodecahedra behaving the $\mathrm{WO}_{4}$ tetrahedra as rigid units [29]. As described above, the different polyhedral contraction causes the anisotropic behaviour of $\mathrm{BaWO}_{4}$. Similar anisotropy is also observed in thermal expansion.

We will discuss now about the crystal structure of the high-pressure phase of $\mathrm{BaWO}_{4}$. Before discussing our results we will briefly describe earlier studies. In XRD experiments performed using silicone oil as PTM, a phase transition from tetragonal scheelite to monoclinic fergusonite was detected at $7.3 \mathrm{GPa}$ [9]. Other XRD experiments carried out using methanol-ethanol-water as PTM reported the phase transition from scheelite-type to monoclinic fergusonite-type at about $7 \mathrm{GPa}$ [10]. A 
second transition to another monoclinic structure was found at $10.9 \mathrm{GPa}$ [9]. The second HP phase was assigned to a structure similar to that of the $\mathrm{BaWO}_{4}$-II structure synthesized under HP-HT conditions $[\mathbf{5}, \mathbf{1 2}, \mathbf{1 3}]$. A more recent x-ray diffraction study performed under HP conditions [3], carried out on quenched $\mathrm{BaWO}_{4}$-II, showed that under similar pressure conditions, the XRD pattern of $\mathrm{BaWO}_{4}-\mathrm{II}$ is different from that of the XRD experiments with silicon oil as PTM [9]. Not only that but also the lattice parameters differ by more than 3\%. Therefore, it was concluded that the phases from the two experiments have different structures and that $\mathrm{BaWO}_{4}$-II cannot be obtained at room temperature by means of simple compression. However, combined large-volume press and Raman experiments [5] have shown that the $\mathrm{BaWO}_{4}$-II phase can be obtained at HP and RT.

In the present case, we have refined the diffraction pattern obtained for the HP phase in the XRD experiment carried out with $\mathrm{Ne}$ as PTM assuming the $\mathrm{BaWO}_{4}$-II phase. The refinement was done by using the structural parameters obtained from single-crystal diffraction experiments [12] as initial values. In the Rietveld refinement, the atomic positions of oxygen atoms were constrained to the original positions. The small x-ray scattering cross-section of oxygen causes uncertainties in the determination of the positions of the oxygen atoms. Consequently, it is not possible to accurately determine twenty four atomic coordinates for different oxygen atoms from the present powder x-ray diffraction experiments. Therefore, only the $\mathrm{Ba}$ and $\mathrm{W}$ positions were refined. After the refinement, we found that the XRD pattern of the HP phase could be well described by the BaWO4-II phase. The refined structure is given in Table III. The small residuals along with the high quality of the Rietveld refinement, and the similitude between the diffraction pattern of the present HP phase and the patterns obtained directly from quenched $\mathrm{BaWO}_{4}$-II support the idea that this structure is 
obtained directly from scheelite in our experiments when $\mathrm{Ne}$ is used as the pressuretransmitting medium. The full structural determination of the $\mathrm{BaWO}_{4}$-II phase needs the performance of high-pressure single-crystal diffraction studies on $\mathrm{BaWO}_{4}$. Such studies have been performed on related compounds [30,31]. We hope the results here reported will trigger similar studies on $\mathrm{BaWO}_{4}$.

It is important to note that according to our structural determination of the HP $\mathrm{BaWO}_{4}$-II structure, the reported phase transition implies an increase of $\mathrm{W}$ coordination from tetrahedral to octahedral. The coordination number of the barium atoms has also increased in comparison with the scheelite structure [12]. On the other hand, there is a relative volume change per formula unit of $-7.1 \%$ at $9.4 \mathrm{GPa}$ from the scheelite-type to the BaWO4-II phase, indicating that the scheelite- $\mathrm{BaWO}_{4}$-II transition is a first-order transformation.

In contrast to the above described results, when no pressure-transmitting medium is used, i.e. under highly non hydrostatic conditions, diffraction patterns from the high-pressure phase are obviously different from those of the $\mathrm{BaWO}_{4}$-II structure as can be seen in Fig. 1b. However, due to the quality of XRD patterns, with very broad peaks caused by non hydrostaticity, the structure of the HP phase cannot be refined in the non-hydrostatic experiments. Apparently, non-hydrostatic stresses can play an important role in the structural sequence of $\mathrm{BaWO}_{4}$, leading to a complex polymorphism. As found in our XRD experiments (and also in our Raman experiments, as we will show latter), at room temperature under quasi-hydrostatic conditions, scheelite $\mathrm{BaWO}_{4}$ transforms directly into monoclinic $\mathrm{BaWO}_{4}$-II beyond 7.6 GPa. This result fully agrees with previous ab initio calculations [9]. The theoretically predicted structural parameters for $\mathrm{BaWO}_{4}$-II agree reasonably well with our experiments [9]. On the other hand, under highly non-hydrostatic conditions, at $8.6 \mathrm{GPa}$ the transition is to a 
structure that has not been resolved because of the broad and strongly overlapped peaks. This could explain the different results that were obtained using other pressure media like silicone oil and methanol-ethanol-water $[9, \mathbf{1 0}]$. In such a case, we have a situation intermediate between the two cases studied here. Therefore, uniaxial stresses usually present when silicone oil and methanol-ethanol-water are used as PTM [32] (which are smaller than without pressure-transmitting medium but higher than in a Ne environment [33]) would lead first to a distortion of scheelite (the monoclinic fergusonite phase) and upon further compression to a monoclinic structure related to $\mathrm{BaWO}_{4}$-II (both have the same space group), which is a distorted version of it. This phase, named before as $\mathrm{BaWO}_{4}$-II [9], should be named differently (we suggest $\beta-\mathrm{BaWO}_{4}$-II) to avoid confusion. The use of a different name will also make clear that $\beta-\mathrm{BaWO}_{4}$-II is not exactly the same structure obtained under HP-HT conditions. Therefore, the $\mathrm{BaWO}_{4}$-II structure apparently can be obtained at RT but only under quasi-hydrostatic conditions. Under such conditions, a possible cause of the direct scheelite- $\mathrm{BaWO}_{4}$-II transition is that pressure lowers the kinetic barrier associated to it [34]. Note that, ab initio calculations [9], identify the $\mathrm{BaWO}_{4}$-II as the structure with the smallest free enthalpy at pressures as low as $3 \mathrm{GPa}$. According with calculations, the transition is direct from scheelite to $\mathrm{BaWO}_{4}$-II with no intermediate phases. The kinetic barrier is what requests the application of $750 \mathrm{~K}$ to obtain the $\mathrm{BaWO}_{4}$-II phase at $4 \mathrm{GPa}$ [5]. However, largevolume press experiments already have shown that the kinetic barrier is reduced upon compression (at $6 \mathrm{GPa}$ only $600 \mathrm{~K}$ are needed). All these facts are consistent with the transformation to $\mathrm{BaWO}_{4}$-II detected here under quasi-hydrostatic conditions beyond 7.6 $\mathrm{GPa}$ at RT. A last comment we would like to make before discussing Raman experiments is that according to the calculations multiple minima are found in the energy landscape of $\mathrm{BaWO}_{4}$. The presence of several minima is consistent with the 
complex polymorphism observed in $\mathrm{BaWO}_{4}$. In particular, uniaxial stresses can favor the transition to the structures associated to these minima, which do not correspond to the thermodynamically stable phase $\left(\mathrm{BaWO}_{4}-\mathrm{II}\right)$. Thus, monoclinic fergusonite and $\beta$ $\mathrm{BaWO}_{4}$-II, as well as the unresolved structure reported here are observed at similar pressures than that of $\mathrm{BaWO}_{4}$-II depending upon experimental conditions and sample history.

\section{B. Raman experiments}

The scheelite structure of $\mathrm{BaWO}_{4}$ is centrosymmetric and has space group $I 4_{1} / \mathrm{a}$ with four formula units per unit cell. Group theoretical considerations lead to 13 zonecenter Raman-active modes [7]

$$
\Gamma=3 A_{g}+5 B_{g}+5 E_{g}
$$

where $A$ and $B$ modes are nondegenerate, and the $E$ modes are doubly degenerate.

Figs. $4 \mathrm{a}$ y $4 \mathrm{~b}$ show a selection of Raman spectra collected at different pressures. At ambient pressure, the Raman spectra can be clearly assigned to the scheelite structure [7, 11]. Twelve out of the thirteen modes are identified. They are listed in Table IV. Fig. 5 shows the evolution of the Raman modes with pressure. The agreement with previous measurements is quite good not only for the Raman frequencies $(\omega)$, but also for the pressure coefficients $(\mathrm{d} \omega / \mathrm{dP})$. Thus, the use of different pressure media apparently does not affect the behavior of the Raman modes as much as it does with the lattice parameters. The undetected mode of scheelite is due to the overlap of one $A_{g}$ and one $B_{g}$ modes near $330 \mathrm{~cm}^{-1}$ [7]. From ambient pressure to $6.0 \mathrm{GPa}$ all the Raman spectra undoubtedly belong to the scheelite structure. The typical soft-mode behavior of the lowest-frequency mode of scheelite is clearly observed. The presence of this mode is related to mechanical instabilities, which drive the pressure-induced transition [35]. 
At 7.2 GPa, additional Raman peaks appear indicating the onset of the transition, but scheelite modes are still present. The transition is completed at $7.6 \mathrm{GPa}$ with the disappearance of the scheelite modes. In Figs. $4 a$ and $4 b$, the differences between the Raman spectra of the low- and high-pressure phases can be clearly seen. At $9.4 \mathrm{GPa}$, we have detected forty Raman modes for the high-pressure phase. They are listed in Table $\mathrm{V}$ and compared with earlier data obtained directly from quenched $\mathrm{BaWO}_{4}-\mathrm{II}$ at a similar pressure [6] and from the post-scheelite phase observed in a previous study [7]. Their pressure coefficients are also listed. As already pointed out by Lacomba-Perales et al. [5], we found a reasonable good agreement between the Raman spectrum obtained for the post-scheelite phase and that obtained directly from $\mathrm{BaWO}_{4}$-II. The agreement of the pressure coefficients is also reasonable. According to group theory, there are seventy-two Raman-active modes in $\mathrm{BaWO}_{4}$-II represented as [7]

$$
\Gamma=36 A_{g}+36 B_{g}
$$

Out of these modes, only twenty-seven modes were reported by Tan et al. [6]. Twenty-one of these modes perfectly match Raman modes here detected. The rest of the modes we found agree quite well with earlier calculations [7] and with the experiments previously reported by Manjon et al. [7]. Therefore, it is quite likely that the $\mathrm{BaWO}_{4}-\mathrm{II}$ is obtained in our experiments directly from scheelite at RT and 7.6 GPa. This result agrees with the conclusions extracted from XRD experiments. Note that Manjon et al. [7] obtained an intermediate fergusonite-type phase in between scheelite and $\mathrm{BaWO}_{4}$-II due to the use of a less hydrostatic environment. This phase is not observed in the present study.

The pressure evolution of the Raman modes of the post-scheelite phase is shown in Fig. 5. In contrast to scheelite, we did not detect any soft mode in the HP phase (see also Table V). The modes with the highest pressure coefficients are those of the high- 
frequency region. In particular, there is only one high-frequency mode with a small pressure coefficient (the mode at $716 \mathrm{~cm}^{-1}$ at $9.4 \mathrm{GPa}$ ) and the mode with the highest pressure coefficient is the one located at $490 \mathrm{~cm}^{-1}$ at $9.4 \mathrm{GPa}$ (see Table V). Note that the energy gap between stretching and bending modes of $\mathrm{BaWO}_{4}$ decreases from about $440 \mathrm{~cm}^{-1}$ at ambient pressure to only $85 \mathrm{~cm}^{-1}$ at $9.4 \mathrm{GPa}$. The latter is a direct proof of the change of coordination of tungsten, from tetrahedral to octahedral, associated to the phase transition [36]. Upon further compression, we did not find any qualitative change in the Raman spectrum of $\mathrm{BaWO}_{4}$-II. The only noticeable facts were, the gradual lost of Raman intensity, the broadening of some peaks (both typical of HP experiments), and the merging (or crossover) of part of the detected Raman modes, in particular beyond 25.2 GPa. Therefore, the phase transition predicted by ab initio calculations to occur at $27 \mathrm{GPa}$ (from BaWO4-II to an orthorhombic structure) [9] cannot be confirmed by our experiments. Additionally, we did not find any hint of the amorphization reported to occur beyond $47 \mathrm{GPa}$ in previous experiments [9] probably due to the Ne pressure medium used in our experiment. Upon pressure release from $44.3 \mathrm{GPa}$, the observed phase transition is reversible (see top Raman spectrum in Fig. 4b) and the scheelite phase is recovered in agreement with our XRD experiments.

\section{Concluding Remarks}

We have performed HP XRD and Raman experiments on $\mathrm{BaWO}_{4}$. We have found that under quasi-hydrostatic conditions, above $7.6 \mathrm{GPa}$ the low-pressure tetragonal scheelite structure transforms at RT directly into the much denser monoclinic $\mathrm{BaWO}_{4}$-II structure. We have also found that non hydrostaticity could induce transitions to different structures, leading to a rich polymorphism in $\mathrm{BaWO}_{4}$. The crystal structure of the low- and high-pressure phases has been refined when Ne is used as PTM. For the low-pressure phase the effects of pressure on bulk and axial compressibilities is 
discussed. In addition, the pressure evolution of the Raman modes of different phases is reported. The results reported here confirm previous theoretical studies using two independent experimental techniques. They are also important for understanding the role of pressure in stabilizing different structures in $\mathrm{ABO}_{4}$ oxides of technological interest. These results are also relevant for geophysics given the close relation between the crystal structure of $\mathrm{BaWO}_{4}$ at ambient pressure and that of zircon. In contrast with our study, previous experiments did not use hydrostatic pressure media, suggesting that non-hydrostaticity may affect the structural transitions. Consequently, the present results will call to revisit all previous studies already performed in scheelite-type oxides upon compression. Another open question for the future are the transition mechanisms driving the different transitions observed in $\mathrm{BaWO}_{4}$ under different pressure environments. The answer of this question will request not only experimental studies but also theoretical calculations like those performed for $\mathrm{ZrSiO}_{4}$ and $\mathrm{AgClO}_{4}[\mathbf{3 7}, \mathbf{3 8}]$.

\section{Acknowledgments}

Research supported by Spanish MEC (Grant No: MAT2010-21270-C04-01/04), MALTA Consolider Ingenio 2010 (CSD2007-00045), and Vicerrectorado de Investigación y Desarrollo of the Universitat Politècnica de València (UPV2011-0914 PAID-05-11 and UPV2011-0966 PAID-06-11). XRD data collected at HPCAT, Advanced Photon Source (APS), Argonne National Laboratory. HPCAT is supported by CIW, CDAC, UNLV and LLNL through funding from DOE-NNSA, DOE-BES, and NSF. APS is supported by DOE-BES under DEAC02-06CH11357. 


\section{References}

* Dr. Oscar Gomis, osgohi@fis.upv.es.

[1] E. Gürmen, E. Daniels, and J.S. King, J. Chem. Physics 55, 1093 (1971).

[2] D. Errandonea and F.J. Manjon, Progress in Materials Science 53, 711 (2008).

[3] D. Tan, W. Xiao, W. Zhou, M. Chen, W. Zhou, X. Li, Y. Li, and J. Liu, To appear in High Pressure Research (2012); DOI:10.1080/08957959.2012.658789.

[4] R. Lacomba-Perales, D. Errandonea, A. Segura, J. Ruiz-Fuertes, P. RodriguezHernandez, S. Radescu, J. Lopez-Solano, A. Mujica, and A. Muñoz, J. Appl. Phys. 110, 043703 (2011).

[5] R. Lacomba-Perales, D. Martinez-Garcia, D. Errandonea, Y. Le Godec, J. Philippe,

G. Le Marchand, J.C. Chervin, A. Polian, A. Muñoz, and J. Lopez-Solano, Phys. Rev. B 81,144117 (2010).

[6] D. Tan, W. Xiao, W. Zhou, M. Song, X. Xiong, and M. Chen, Chin. Phys. Lett. 26, 046301 (2009).

[7] F.J. Manjon, D. Errandonea, N. Garro, J. Pellicer-Porres, P. Rodriguez-Hernandez, S. Radescu, J. Lopez-Solano, A. Mujica, and A. Muñoz, Phys. Rev. B 74, 144111 (2006).

[8] A. Grzechnik, W. A. Chrichton, W. G. Marshall, and K. Friese, J. Phys.: Condens. Matter 18, 3017 (2006).

[9] D. Errandonea, J. Pellicer-Porres, F.J. Manjon, A. Segura, Ch. Ferrer-Roca, R.S. Kumar, O. Tschauner, J. Lopez-Solano, P. Rodriguez-Hernandez, S. Radescu, A. Mujica, A. Muñoz, and G. Aquilanti, Phys. Rev. B 73, 224103 (2006).

[10] V. Panchal, N. Garg, A.K. Chauhan, S. Sangeeta, and S.M. Sharma, Solid State Commun. 130, 203 (2004).

[11] A. Jayaraman, B. Batlogg, and L.G. Van Uitert, Phys. Rev. B 28, 4774 (1983). 
[12] I. Kawada, K. Kato, and T. Fujita, Acta Cryst. B 30, 2069 (1974).

[13]. T. Fujita, S. Yamaoka, and O. Fukunaga, Mater. Research Bull. 9, 141 (1974).

[14] F.J. Manjon, D. Errandonea, N. Garro, J. Pellicer-Porres, J. Lopez-Solano, P. Rodriguez-Hernandez, S. Radescu, , A. Mujica, and A. Muñoz, Phys. Rev. B 74, $144112(2006)$.

[15] D. Errandonea, D. Martínez-García, R. Lacomba-Perales, J. Ruiz-Fuertes, and A. Segura, Appl. Phys. Lett. 89, 091913 (2006).

[16] H. K. Mao, J. Xu, and P. M. Bell, J. Geophys. Res. 91, 4673 (1986).

[17] S. Klotz, J.C. Chervin, P. Munsch, and G. Le Marchand, J. Phys. D: Appl. Phys. 42, 075413 (2009).

[18] A. P. Hammersley, S. O. Svensson, M. Hanfland, A. N. Fitch, and D. Häusermann, High Pressure Research 14, 235 (1996).

[19] T. J. B. Holland, and S. A. T. Redfern, Mineralogical Magazine, 61, 65 (1997).

[20] A. C. Larson and R. B. von Dreele, LANL Report 86-748, 2004 (unpublished).

[21] B. H. Toby, J. Appl. Cryst. 34, 210 (2001).

[22] W. Kraus and G. Nolze, J. Appl. Crystallogr. 29, 301 (1996).

[23] F. Birch, J. Geophys. Res. 83, 1257 (1978).

[24] H. Liu, Y. Ding, M. Somayazulu, J. Qian, J. Shu, D. Häusermann, and H.-K. Mao, Phys. Rev. B 71, 212103 (2005).

[25] H. Liu, J. Hu, J. Shu, D. Häusermann, and H.-K. Mao, Appl. Phys. Lett. 85, 1973 (2004).

[26] J. Ruiz-Fuertes, D. Errandonea, R. Lacomba-Perales, A. Segura, J. González, F. Rodríguez, F. J. Manjón, S. Ray, P. Rodríguez-Hernández, A. Muñoz, Zh. Zhu, and C. Y. Tu, Phys. Rev. B 81, 224115 (2010). 
[27] D. Santamaría-Pérez, L. Gracia, G. Garbarino, A. Beltrán, R. Chulia-Jordan, O.

Gomis, D. Errandonea, Ch. Ferrer-Roca, D. Martínez-García, and A. Segura, Phys. Rev. B 84, 054102 (2011).

[28] L.W. Finger, M. Kroeker, and B.H. Toby, J. Applied Cryst. 40, 188 (2007).

[29] S.N. Achary, S.J. Patwe, M.D. Mathews, A.K. Tyagi, Journal of Physics and Chemistry of Solids 67, 774 (2006).

[30] D. Machon, V. P. Dmitriev, P. Bouvier, P. N. Timonin, V. B. Shirokov, and H.-P. Weber, Phys. Rev. B 68, 144104 (2003).

[31] J. Ruiz-Fuertes, A. Friedrich, J. Pellicer-Porres, D. Errandonea, A. Segura, W. Morgenroth, E. Haussühl, C.-Y. Tu, and A. Polian, Chem. Mater. 23, 4220 (2011).

[32] D. Errandonea, Y. Meng, M. Somayazulu, and D. Häusermann, Physica B 355, $116(2005)$.

[32] D. Errandonea, R. S. Kumar, J. Ruiz-Fuertes, A. Segura, and E. Haussühl, Phys. Rev. B 83, 144104 (2011).

[34] J. T. Wang, C. Chen, and Y. Kawazoe, Phys. Rev. Letters 106, 075501 (2011).

[35] D. Errandonea and F. J. Manjon, Materials Research Bulletin 44, 807 (2009).

[36] M. Maczka, A. G. Souza Filho, W. Paraguassu, P. T. C. Freire, J. Mendes Filho, and J. Hanuza, Progress in Materials Science 57, 1335 (2012).

[37] M. Flórez, J. Contreras-García, J. M. Recio, and M. Marqués, Phys. Rev. B 79, 104101 (2009).

[38] D. Errandonea, L. Gracia, A. Beltrán, A. Vegas, and Y. Meng, Phys. Rev. B 84, 064103 (2011). 
Table I: Structural parameters of scheelite-type $\mathrm{BaWO}_{4}$ at different pressures. Atomic coordinates are given with origin choice 2 for space group $I 4_{1} / a$.

Ambient pressure [1]: $a=5.6134 \AA, c=12.72 \AA$

\begin{tabular}{ccccc}
\hline \hline & Site & $\mathrm{X}$ & $\mathrm{y}$ & $\mathrm{z}$ \\
\hline $\mathrm{Ba}$ & $4 \mathrm{~b}$ & 0 & 0.25 & 0.625 \\
$\mathrm{~W}$ & $4 \mathrm{a}$ & 0 & 0.25 & 0.125 \\
$\mathrm{O}$ & $16 \mathrm{f}$ & 0.1227 & 0.01680 & 0.2031 \\
\hline \hline
\end{tabular}

1.0 GPa: $a=5.603(2) \AA, c=12.693(8) \AA, R_{p}=3.3 \%, R_{w p}=7.9 \%$. Ne used as pressure transmitting medium.

\begin{tabular}{ccccc}
\hline & Site & $\mathrm{X}$ & $\mathrm{y}$ & $\mathrm{z}$ \\
\hline $\mathrm{Ba}$ & $4 \mathrm{~b}$ & 0 & 0.25 & 0.625 \\
$\mathrm{~W}$ & $4 \mathrm{a}$ & 0 & 0.25 & 0.125 \\
$\mathrm{O}$ & $16 \mathrm{f}$ & $0.1232(4)$ & $0.0174(4)$ & $0.2033(2)$ \\
\hline \hline
\end{tabular}

7.6 GPa: $a=5.437(2) \AA, c=12.060(7) \AA, R_{p}=3.5 \%, R_{w p}=8.3 \%$. Ne used as pressure transmitting medium.

\begin{tabular}{ccccc}
\hline \hline & Site & $\mathrm{X}$ & $\mathrm{y}$ & $\mathrm{z}$ \\
\hline $\mathrm{Ba}$ & $4 \mathrm{~b}$ & 0 & 0.25 & 0.625 \\
$\mathrm{~W}$ & $4 \mathrm{a}$ & 0 & 0.25 & 0.125 \\
$\mathrm{O}$ & $16 \mathrm{f}$ & $0.1276(3)$ & $0.0224(3)$ & $0.2055(2)$ \\
\hline \hline
\end{tabular}


Table II: EOS parameters and axial compressibilities $\left(\kappa_{x}=\frac{-1}{x} \frac{\partial x}{\partial P}\right)$ at ambient pressure of scheelite-type $\mathrm{BaWO}_{4}$ obtained under different pressure-transmitting media (PTM). The axial compressibilities were obtained assuming a quadratic evolution for the lattice parameters.

\begin{tabular}{|c|c|c|c|c|c|c|}
\hline PTM & $\begin{array}{l}V_{0} \\
\left(\AA^{3}\right)\end{array}$ & $\begin{array}{c}\mathrm{B}_{0} \\
(\mathrm{GPa})\end{array}$ & $\mathrm{B}_{0}{ }^{\prime}$ & $\begin{array}{c}\kappa_{a} \\
\left(10^{-3} \mathrm{GPa}^{-1}\right)\end{array}$ & $\begin{array}{c}\kappa_{c} \\
\left(10^{-3} \mathrm{GPa}^{-1}\right)\end{array}$ & $\kappa_{c} / \kappa_{a}$ \\
\hline $\mathrm{Ne}$ & 404.1 & $47(2)$ & 4 & $5.9(2)$ & $12.4(5)$ & $2.1(2)$ \\
\hline $\begin{array}{l}\text { Methanol- } \\
\text { ethanol - } \\
\text { water [10] }\end{array}$ & $\begin{array}{l}404.1 \\
404.1\end{array}$ & $\begin{array}{c}57 \\
54(2)\end{array}$ & $\begin{array}{c}3.5 \\
4\end{array}$ & $4.9(3)$ & $7.7(4)$ & $1.6(2)$ \\
\hline Silicon oil [9] & $\begin{array}{c}402.8(9) \\
402.8\end{array}$ & $\begin{array}{l}52(5) \\
55(5)\end{array}$ & $\begin{array}{c}5(1) \\
4\end{array}$ & $5.4(5)$ & $9(1)$ & $1.8(3)$ \\
\hline Without PTM & 401.9(3) & $66(4)$ & $4(2)$ & $4.3(2)$ & $6.4(8)$ & $1.5(2)$ \\
\hline
\end{tabular}


Table III: Structural parameters of $\mathrm{BaWO}_{4}$-II at 9.4 GPa. $a=12.628(9), \quad b=6.949(5)$, $c=7.39(2), \beta=91.20(8) . R_{p}=2.1 \%, R_{w p}=4.3 \%$. Ne used as pressure transmitting medium. Oxygen positions are fixed to those given in Ref. [12].

\begin{tabular}{ccccc}
\hline \hline & Site & $\mathrm{X}$ & $\mathrm{y}$ & $\mathrm{z}$ \\
\hline $\mathrm{Ba}_{1}$ & $4 \mathrm{e}$ & $0.162(1)$ & $0.652(2)$ & $0.161(2)$ \\
$\mathrm{Ba}_{2}$ & $4 \mathrm{e}$ & $0.138(2)$ & $0.954(1)$ & $0.630(1)$ \\
$\mathrm{W}_{1}$ & $4 \mathrm{e}$ & $0.084(2)$ & $0.161(2)$ & $0.084(2)$ \\
$\mathrm{W}_{2}$ & $4 \mathrm{e}$ & $0.091(2)$ & $0.459(1)$ & $0.650(1)$ \\
$\mathrm{O}_{1}$ & $4 \mathrm{e}$ & $0.1091(16)$ & $0.0245(32)$ & $0.2972(29)$ \\
$\mathrm{O}_{2}$ & $4 \mathrm{e}$ & $0.1745(16)$ & $0.5851(32)$ & $0.7973(29)$ \\
$\mathrm{O}_{3}$ & $4 \mathrm{e}$ & $0.0524(16)$ & $0.6357(33)$ & $0.4705(28)$ \\
$\mathrm{O}_{4}$ & $4 \mathrm{e}$ & $0.2110(16)$ & $0.2530(32)$ & $0.0571(29)$ \\
$\mathrm{O}_{5}$ & $4 \mathrm{e}$ & $0.0499(16)$ & $0.2709(32)$ & $0.8235(29)$ \\
$\mathrm{O}_{6}$ & $4 \mathrm{e}$ & $0.1716(16)$ & $0.3095(32)$ & $0.5222(29)$ \\
$\mathrm{O}_{7}$ & $4 \mathrm{e}$ & $0.0219(16)$ & $0.3651(33)$ & $0.1887(29)$ \\
$\mathrm{O}_{8}$ & $4 \mathrm{e}$ & $0.0740(16)$ & $0.9304(33)$ & $0.9467(29)$ \\
\hline \hline
\end{tabular}


Table IV: Raman modes and pressure coefficients for the scheelite-type phase.

\begin{tabular}{c|cc|cc|cc}
\hline \hline & \multicolumn{2}{|c|}{ This work } & \multicolumn{2}{|c|}{ Manjon et al. [7] } & \multicolumn{2}{|c}{ Jayaraman et al. [11] } \\
\hline Mode & $\omega\left(\mathrm{cm}^{-1}\right)$ & $\begin{array}{c}\mathrm{d} \omega / \mathrm{dP} \\
\left(\mathrm{cm}^{-1} / \mathrm{GPa}\right)\end{array}$ & $\omega\left(\mathrm{cm}^{-1}\right)$ & $\begin{array}{c}\mathrm{d} \omega / \mathrm{dP} \\
\left(\mathrm{cm}^{-1} / \mathrm{GPa}\right)\end{array}$ & $\omega\left(\mathrm{cm}^{-1}\right)$ & $\begin{array}{c}\mathrm{d} \omega / \mathrm{dP} \\
\left(\mathrm{cm}^{-1} / \mathrm{GPa}\right)\end{array}$ \\
\hline$B_{g}$ & 63 & -1.1 & 63 & -0.8 & 62 & -1.0 \\
$E_{g}$ & 74 & 0.9 & 74 & 1.0 & 74 & 0.8 \\
$E_{g}$ & 101 & 3.3 & 101 & 3.3 & 104 & 0.0 \\
$B_{g}$ & 131 & 4.1 & 133 & 4.1 & & \\
$A_{g}$ & 150 & 4.2 & 150 & 4.2 & 150 & 4.4 \\
$E_{g}$ & 191 & 6.6 & 191 & 6.3 & & \\
$A_{g}$ & & & 331 & 2.5 & & 3.1 \\
$B_{g}$ & 333 & 2.9 & 332 & 3.0 & 332 & 2.0 \\
$B_{g}$ & 344 & 2.7 & 344 & 2.0 & 345 & \\
$E_{g}$ & 353 & 2.9 & 352 & 3.4 & & 3.2 \\
$E_{g}$ & 795 & 3.4 & 795 & 3.2 & 796 & 2.2 \\
$B_{g}$ & 831 & 2.1 & 831 & 2.0 & 830 & 2.8 \\
$A_{g}$ & 925 & 2.9 & 926 & 2.7 & 925 & \\
\hline \hline
\end{tabular}


Table V: Raman modes and pressure coefficients for $\mathrm{BaWO}_{4}$-II phase. The coefficients are obtained from a linear fit. Frequencies are given at the indicated pressures.

\begin{tabular}{|c|c|c|c|c|c|}
\hline \multicolumn{2}{|c|}{$\begin{array}{l}\text { This work } \\
9.4 \mathrm{GPa}\end{array}$} & \multicolumn{2}{|c|}{$\begin{array}{l}\text { Manjon et al. [7] } \\
9 \mathrm{GPa}\end{array}$} & \multicolumn{2}{|c|}{$\begin{array}{l}\text { Tan et al. [6] } \\
8.9 \mathrm{GPa}\end{array}$} \\
\hline$\omega\left(\mathrm{cm}^{-1}\right)$ & $\begin{array}{c}\mathrm{d} \omega / \mathrm{dP} \\
\left(\mathrm{cm}^{-1} / \mathrm{GPa}\right)\end{array}$ & $\omega\left(\mathrm{cm}^{-1}\right)$ & $\begin{array}{c}\mathrm{d} \omega / \mathrm{dP} \\
\left(\mathrm{cm}^{-1} / \mathrm{GPa}\right)\end{array}$ & $\omega\left(\mathrm{cm}^{-1}\right)$ & $\begin{array}{c}\mathrm{d} \omega / \mathrm{dP} \\
\left(\mathrm{cm}^{-1} / \mathrm{GPa}\right)\end{array}$ \\
\hline 62 & 1.1 & & & & \\
\hline 70 & 0.6 & 71 & 0.3 & & \\
\hline 81 & 1.1 & & & & \\
\hline 100 & 1.0 & 98 & 0.5 & & \\
\hline 131 & 1.0 & 130 & 1.2 & & \\
\hline 161 & 2.5 & 152 & 2.4 & & \\
\hline \multirow[t]{2}{*}{169} & 2.4 & 164 & 2.6 & 159 & 3.0 \\
\hline & & 181 & 2.6 & 183 & 3.8 \\
\hline \multirow[t]{2}{*}{195} & 1.9 & & & 197 & 1.9 \\
\hline & & 209 & 2.5 & & \\
\hline \multirow[t]{2}{*}{218} & 1.9 & 218 & 1.7 & 219 & 2.0 \\
\hline & & 234 & 2.7 & & \\
\hline 238 & 1.9 & & & 242 & 1.9 \\
\hline \multirow[t]{2}{*}{262} & 2.5 & 258 & 3.1 & & \\
\hline & & & & 276 & 1.6 \\
\hline \multirow[t]{2}{*}{284} & 2.0 & 287 & 1.7 & 280 & 3.0 \\
\hline & & & & 295 & 2.9 \\
\hline \multirow[t]{3}{*}{308} & 2.4 & 302 & 2.6 & 308 & 2.6 \\
\hline & & 304 & 0.5 & & \\
\hline & & 326 & 2.0 & & \\
\hline \multirow[t]{2}{*}{338} & 2.3 & 330 & 2.8 & & \\
\hline & & & & 341 & 0.2 \\
\hline 361 & 1.9 & 359 & 2.5 & 359 & 1.3 \\
\hline 373 & 2.0 & 369 & 2.1 & 376 & 1.7 \\
\hline 392 & 1.7 & 400 & 1.7 & & \\
\hline 410 & 2.4 & 404 & 1.8 & 402 & 1.8 \\
\hline \multirow[t]{2}{*}{423} & 2.9 & 417 & 2.6 & 421 & 2.0 \\
\hline & & 432 & 3.2 & & \\
\hline 436 & 2.5 & 435 & 4.3 & 435 & 2.7 \\
\hline \multirow[t]{2}{*}{458} & 2.7 & & & & \\
\hline & & 478 & 2.9 & 469 & 3.5 \\
\hline 490 & 3.9 & 495 & 4.0 & & \\
\hline 515 & 2.8 & & & 509 & 2.8 \\
\hline \multirow[t]{2}{*}{533} & 2.4 & 532 & 2.3 & & \\
\hline & & 546 & 4.3 & 549 & 1.5 \\
\hline \multirow[t]{2}{*}{618} & 2.5 & 614 & 2.1 & & \\
\hline & & 634 & 2.1 & & \\
\hline 650 & 2.3 & 658 & 1.5 & 655 & 3.7 \\
\hline 670 & 3.0 & 673 & 0.5 & 667 & 3.5 \\
\hline 704 & 2.4 & 697 & 2.6 & & \\
\hline 716 & 0.4 & & & 715 & 0.4 \\
\hline 745 & 2.8 & 745 & 2.4 & 757 & 2.7 \\
\hline 786 & 2.5 & 770 & 3.5 & 776 & 2.8 \\
\hline 798 & 3.5 & 798 & 3.5 & & \\
\hline 818 & 3.4 & 815 & 3.9 & 820 & 3.8 \\
\hline 832 & 2.2 & & & & \\
\hline 846 & 2.4 & 843 & 2.4 & 858 & 3.6 \\
\hline 879 & 2.6 & 877 & 2.6 & & \\
\hline 909 & 3.0 & 906 & 3.2 & 903 & 2.8 \\
\hline 927 & 2.8 & 923 & 2.8 & & \\
\hline 948 & 2.7 & 950 & 3.2 & 942 & 3.1 \\
\hline 969 & 3.0 & 963 & 2.9 & & \\
\hline
\end{tabular}




\section{Figure captions}

Figure 1: Selection of XRD patterns collected using $\mathrm{Ne}$ as pressure-transmitting medium (a) and without pressure-transmitting medium (b). In (a) Rietveld refinements are shown for the scheelite-type structure at 1.0 and $7.6 \mathrm{GPa}$ and for the HP phase at 9.4 GPa. Residuals are also shown as solid lines. In (b) the refinement and residuals are only included for the scheelite-type at 1 bar. In the latter case the numerical value for the residuals is $R_{p}=12 \%$ and $R_{w p}=17 \%$. Vertical ticks indicate the position of Bragg reflections. Reflections of $\mathrm{Ne}$ are also shown in (a). In the case of the Rietveld refinements experimental data are plotted as solid circles and the calculated diffractograms as solid lines. In all of the cases the background has been subtracted.

Figure 2: (color online) Pressure evolution of the lattice parameters, unit-cell volume, and cation-oxygen distances obtained from different experiments using different pressure-transmitting media. Solid blue diamonds and red circles refer to our experiments carried out with Ne as PTM and without PTM, respectively. Open squares refer to data with silicon oil as PTM from Ref. [9]. Black up triangles refer to data with methanol-ethanol-water as PTM from Ref. [10]. Magenta down triangles shown in the volume-pressure plot refer to data with h-BN as PTM from Ref. [5]. Ab initio data for cation-oxygen distances taken from Ref. [9] are included as solid lines.

Figure 3: Polyhedral volume of the $\mathrm{WO}_{4}$ tetrahedron and $\mathrm{BaO}_{8}$ bisdisphenoid as a function of pressure. Symbols: experimental data. Lines are just a guide to the eye.

Figure 4: (a) Room temperature Raman scattering spectra of $\mathrm{BaWO}_{4}$ from 1 bar up to 7.6 GPa. At 7.6 GPa the Raman peak positions for the high pressure phase are indicated 
with vertical ticks. The spectrum collected at 7.6 is magnified (x2) in order to facilitate phonon identification. Peaks observed below $50 \mathrm{~cm}^{-1}$ are only observed at $7.6 \mathrm{GPa}$ and therefore have not been assigned to the sample. (b) Room temperature Raman scattering spectra of $\mathrm{BaWO}_{4}$ from 7.6 up to $44.3 \mathrm{GPa}$. The Raman spectrum at $0.1 \mathrm{GPa}$ after releasing pressure is also shown. Background has been subtracted.

Figure 5: Pressure dependence of the Raman modes of $\mathrm{BaWO}_{4}$. Solid and open symbols refer to data from the low- and high-pressure phases of $\mathrm{BaWO}_{4}$, respectively. Solid lines connecting measured data points are shown as a guide to the eyes. 
Figure 1(a)

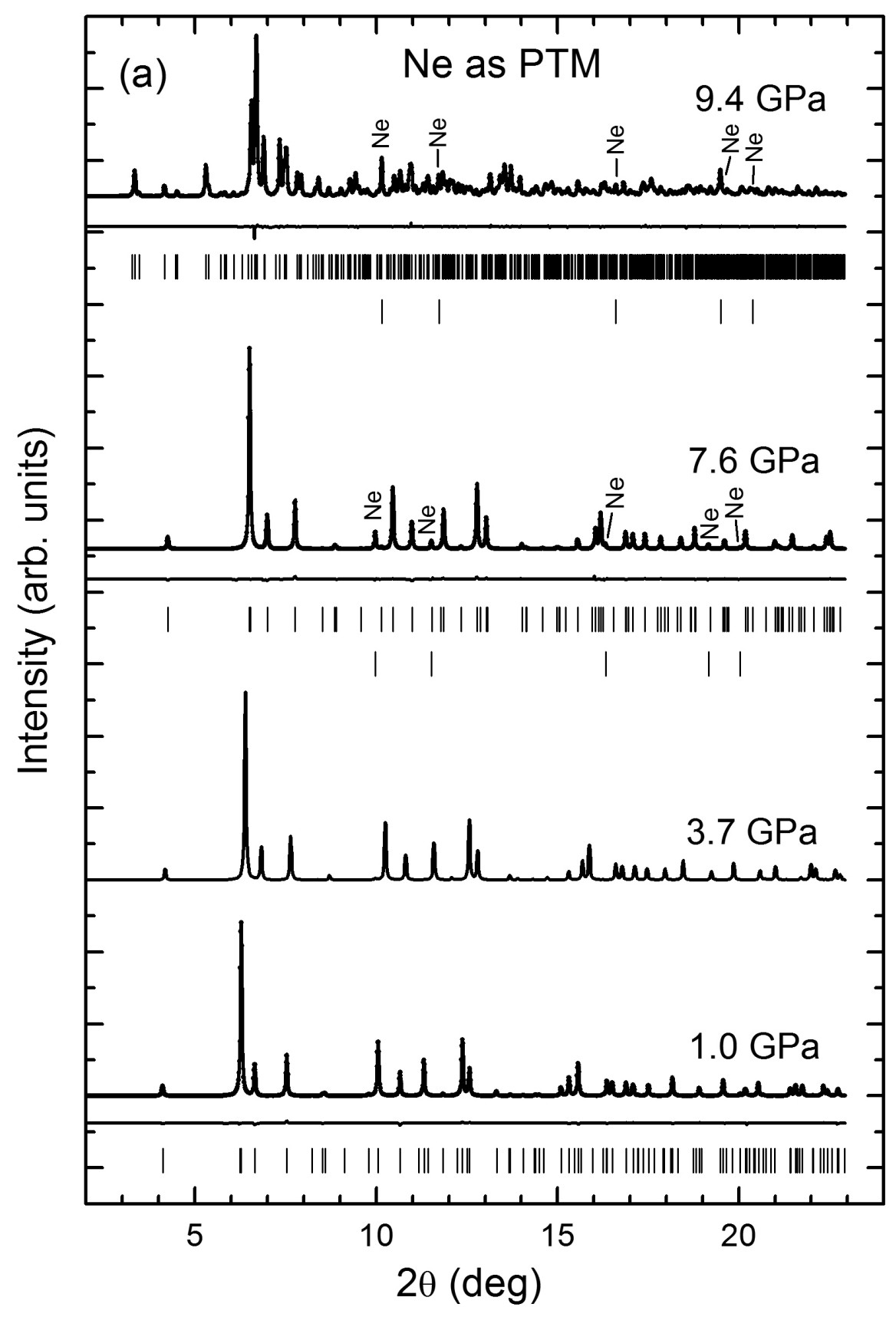


Figure 1(b)

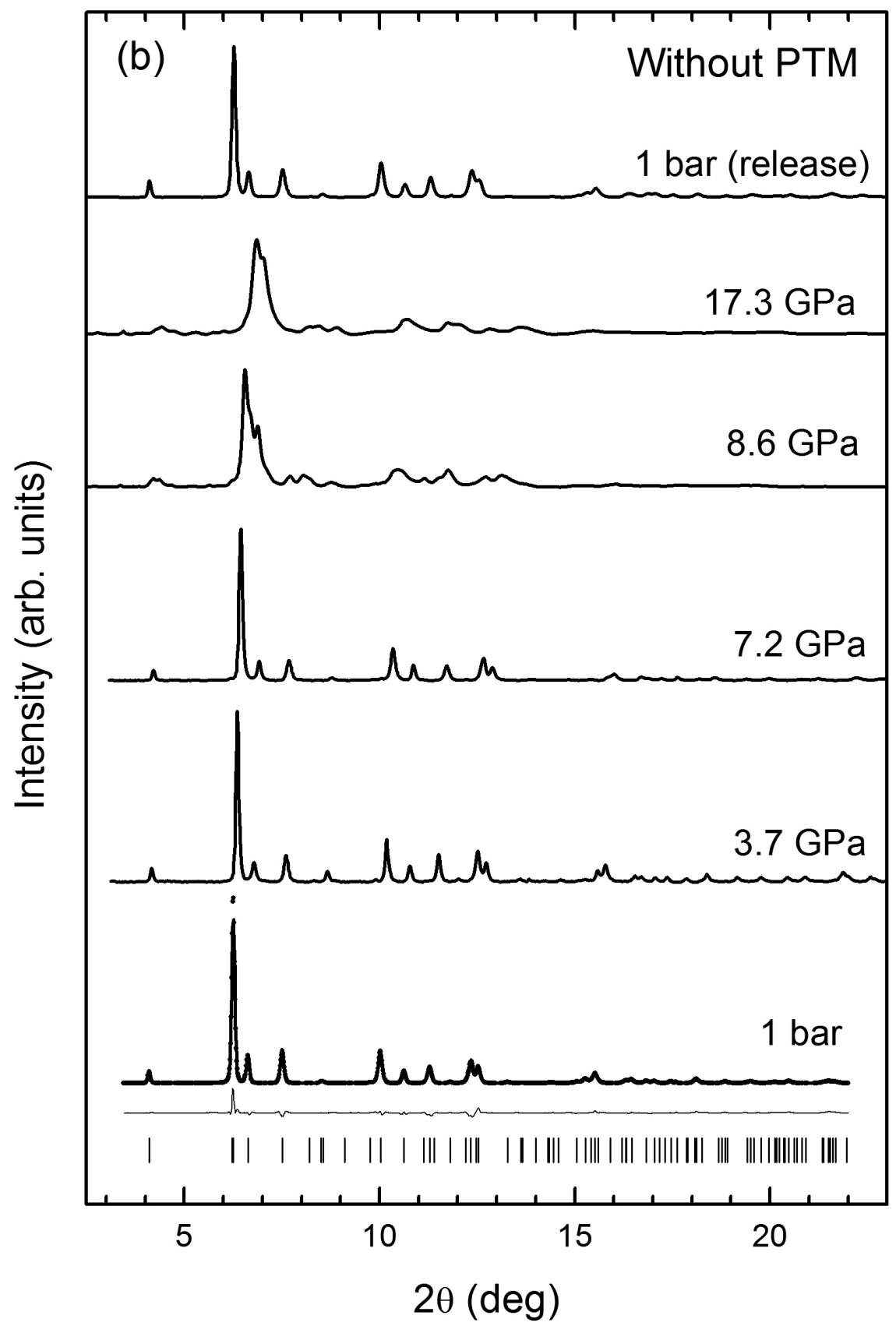


Figure 2
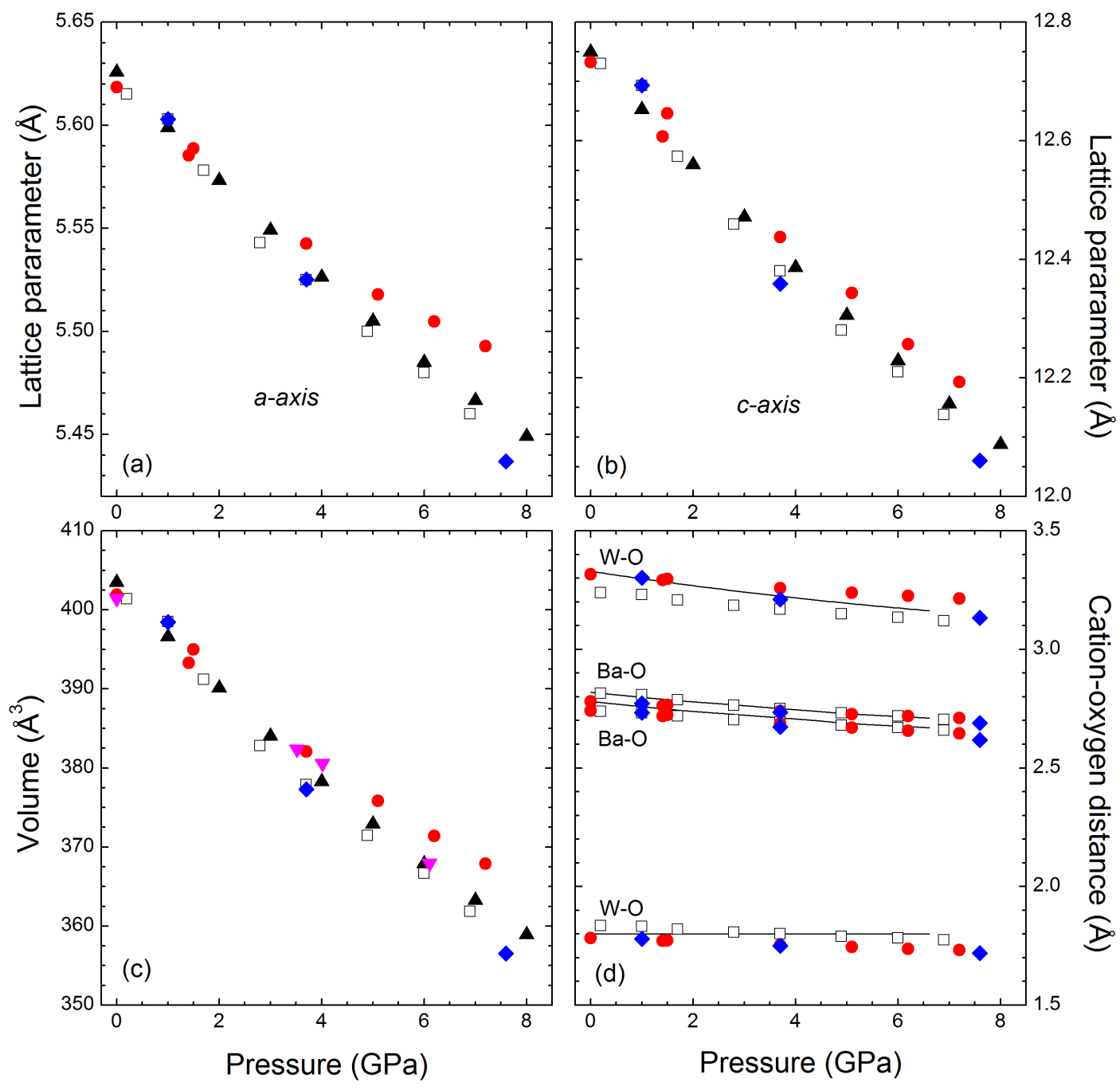
Figure 3

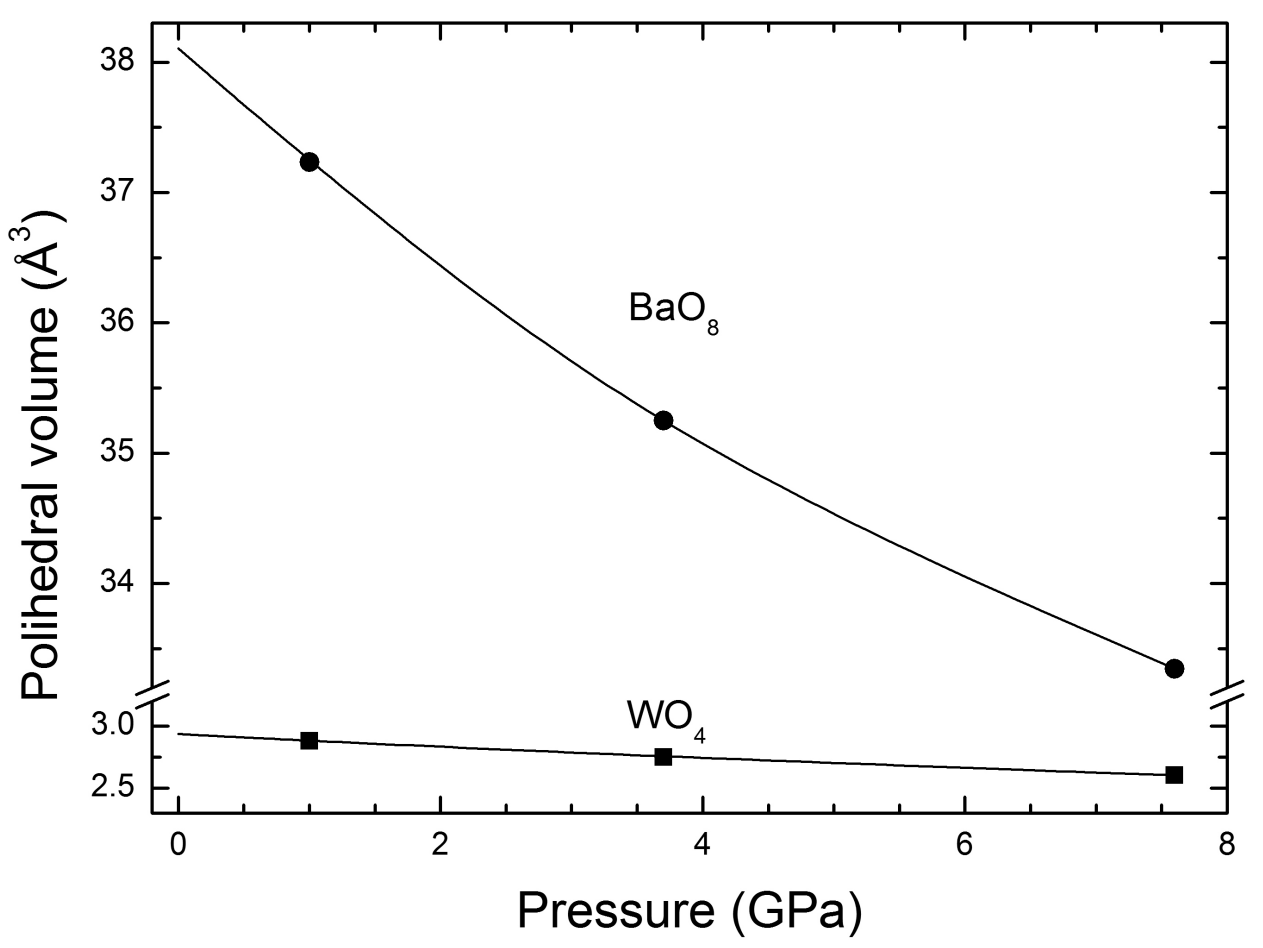


Figure 4(a)

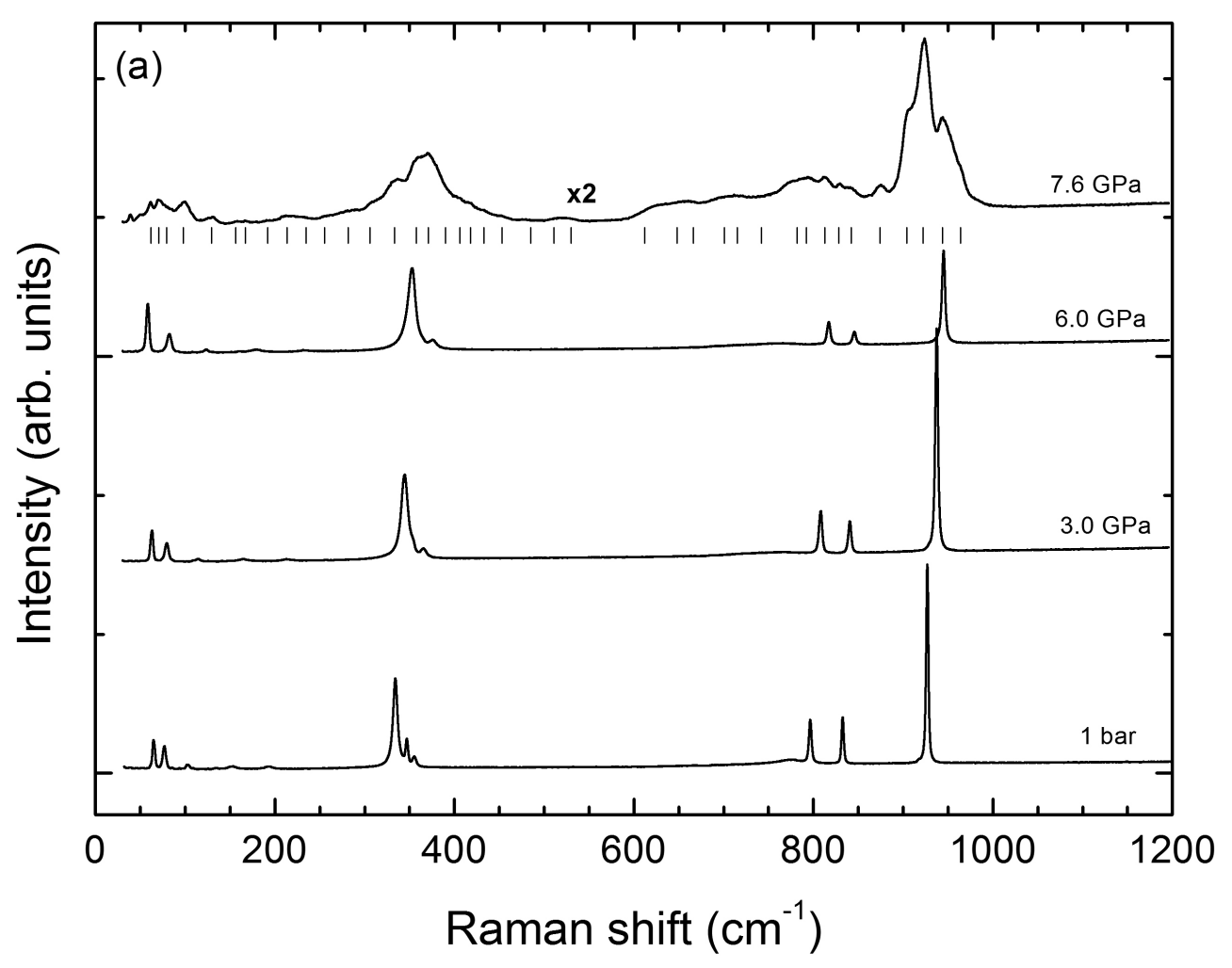


Figure 4(b)

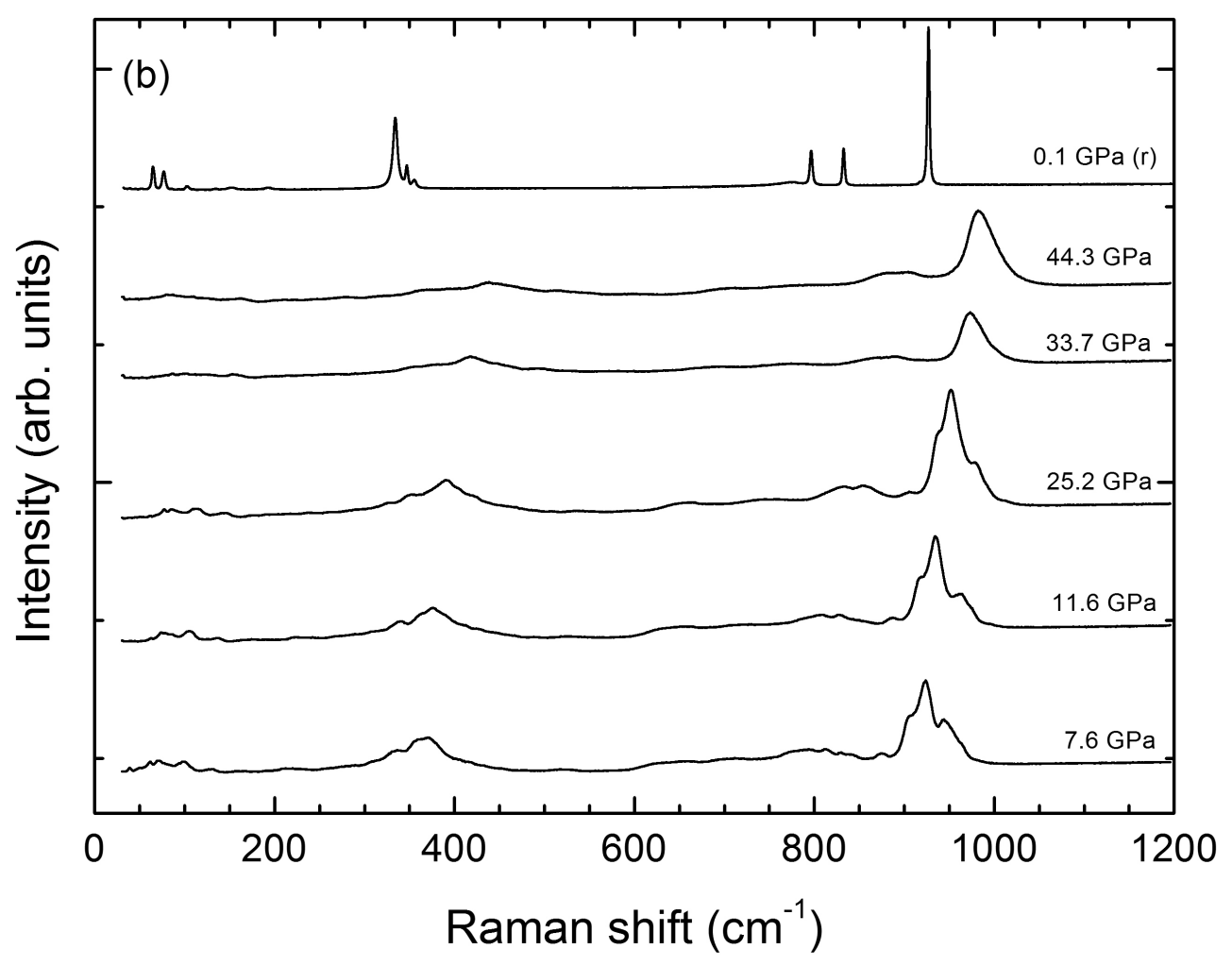


Figure 5

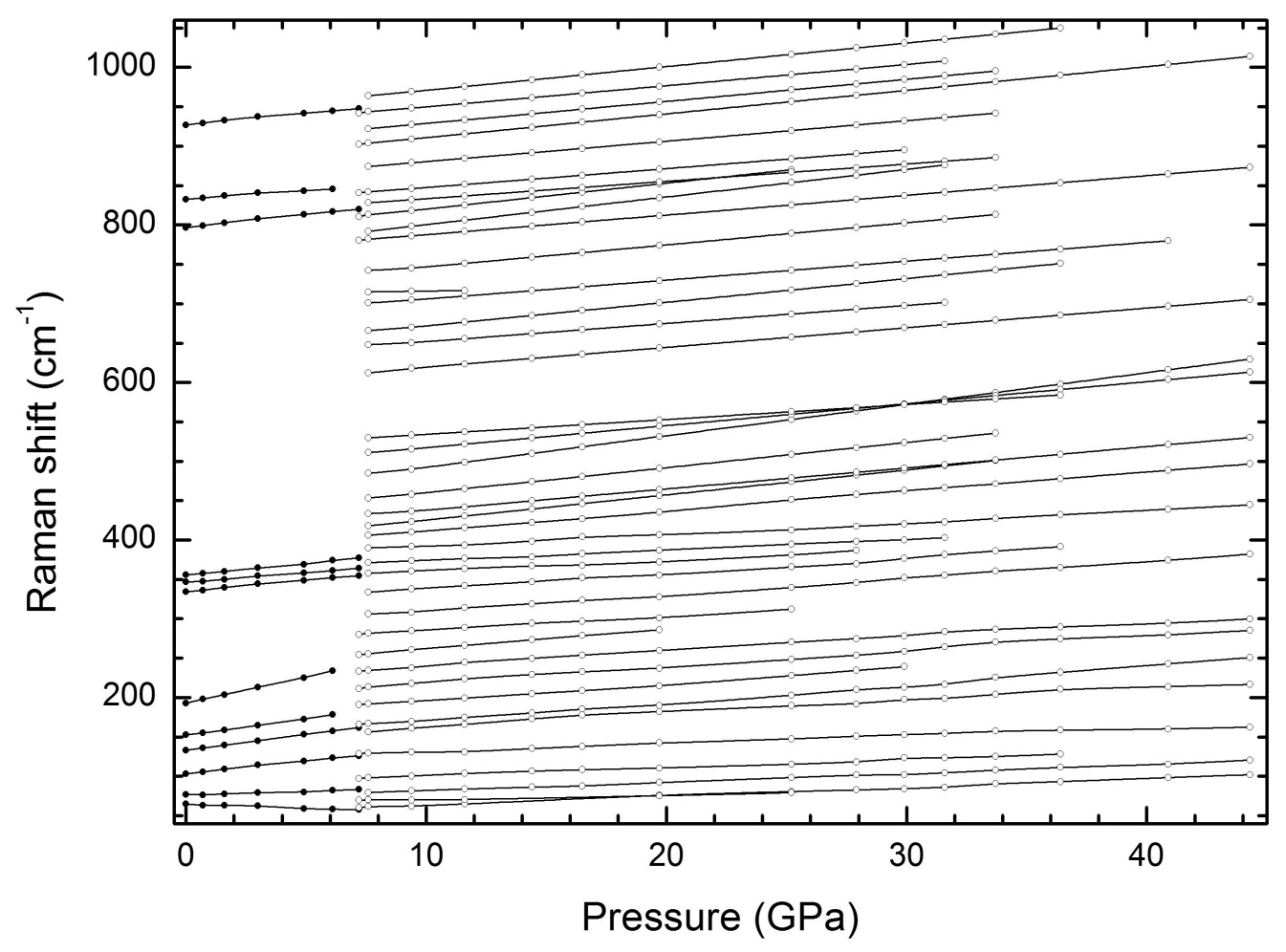

